\title{
Calculations of HREM Image Intensity Using Monte Carlo Integration
}

\author{
L. Y. Chang, R. R. Meyer, A. I. Kirkland \\ Department of Materials, University of Oxford, Parks Road, Oxford, UK. OX1 3PH
}

In order to achieve direct interpretability of HREM images, indirect image restoration methods [1-3] can be used to recover the electron wave function at the specimen exit surface. Most image restoration algorithms involve the linear imaging approximation [4,5], which has the advantage of computational efficiency. Algorithms dealing directly with general nonlinear image intensities, such as maximum likelihood algorithm [6,7], involve minimizing the difference between predicted and experimental image intensities. The computation for these algorithms can therefore be extremely demanding and hence some approximations regarding partial coherence effects have been introduced making these algorithms computationally practical. Here we aim to develop a general and efficient method for image intensity calculation which is suitable for nonlinear image restoration algorithms.

For an incoherent effective source, image intensities are calculated as a weighted summation of coherent image intensities over a range of incident directions (beam tilts), $\boldsymbol{q}$ and defoci, $f$ [8]:

$I(\boldsymbol{r})=\sum_{q} \sum_{f} S(\boldsymbol{q}) F(f) \mid \mathscr{F}^{-1}\left\{\left.\psi(\boldsymbol{k}) a(\boldsymbol{k}) \exp (-2 \pi i \chi(\boldsymbol{k})\}\right|^{2}\right.$

where $S(\boldsymbol{q})$ is the beam divergence distribution, $F(f)$ the focal spread distribution, $\psi(\boldsymbol{k})$ the Fourier transform of the specimen exit wave function, $a(\boldsymbol{k})$ the aperture function and $\chi(\boldsymbol{k})$ the lens aberration function. An approximation can also be made by taking the Taylor expansion of the lens aberration to first order in $\boldsymbol{q}$ and $f$ if the beam tilt $\boldsymbol{q}$ and focal spread $f$ are small, and Equation (1) can then be calculated analytically [9] as:

$$
\begin{aligned}
& I(\boldsymbol{k})=\sum_{\boldsymbol{k}} \psi\left(\boldsymbol{k}+\boldsymbol{k}^{\prime}\right) \psi\left(\boldsymbol{k}^{\prime}\right) T\left(\boldsymbol{k}+\boldsymbol{k}^{\prime}, \boldsymbol{k}^{\prime}\right) \\
& T\left(\boldsymbol{k}_{1}, \boldsymbol{k}_{2}\right)=\exp \left(-i 2 \pi\left[\chi\left(\boldsymbol{k}_{1}\right)-\chi\left(\boldsymbol{k}_{2}\right)\right]\right) \exp \left(-\pi^{2} q_{0}^{2}\left[\nabla \chi\left(\boldsymbol{k}_{1}\right)-\nabla \chi\left(\boldsymbol{k}_{2}\right)\right]^{2}\right) \exp \left(-\pi^{2} \Delta^{2}\left[\partial_{C_{1}} \chi\left(\boldsymbol{k}_{1}\right)-\partial_{C_{1}} \chi\left(\boldsymbol{k}_{2}\right)\right]^{2}\right)
\end{aligned}
$$

where $T$ is the transmission cross coefficient for a strong object. Equation (2) involves $\mathrm{N}^{4}$ operations for a $\mathrm{N} \times \mathrm{N}$ pixel image, which is computationally impractical.

To improve the efficiency and accuracy of image intensity calculation for a general object given by Equation (1), a Monte Carlo integration approach [10] is applied. The sampling points $\boldsymbol{q}$ and $f$ are randomly distributed with their probability chosen according to $S(\boldsymbol{q})$ and $F(f)$. Besides its efficiency, a significant advantage of Monte Carlo integration is that, due to the random sampling, any artefact introduced is random rather than systematic. This is particularly beneficial for nonlinear image restoration algorithms, such as the maximum-likelihood method using a series of images. The accuracy of the Monte Carlo approach is compared by calculating the root-mean-square (RMS) difference between the image spectrum calculated using the Monte Carlo method and that calculated using the TCC for strong objects, as a function of the number of sampling points, shown in Fig. 1 and 2. The results show that the number of the sampling points required was found to be only weakly dependent on atomic number, defocus and thickness, which demonstrates the reliability and general applicability of the method. In practice, only 100 sampling points are required to apply this approach as its RMS difference is about $10^{-2}$, which is smaller than typical experimental noise levels of $6-15 \%$ [11]. 


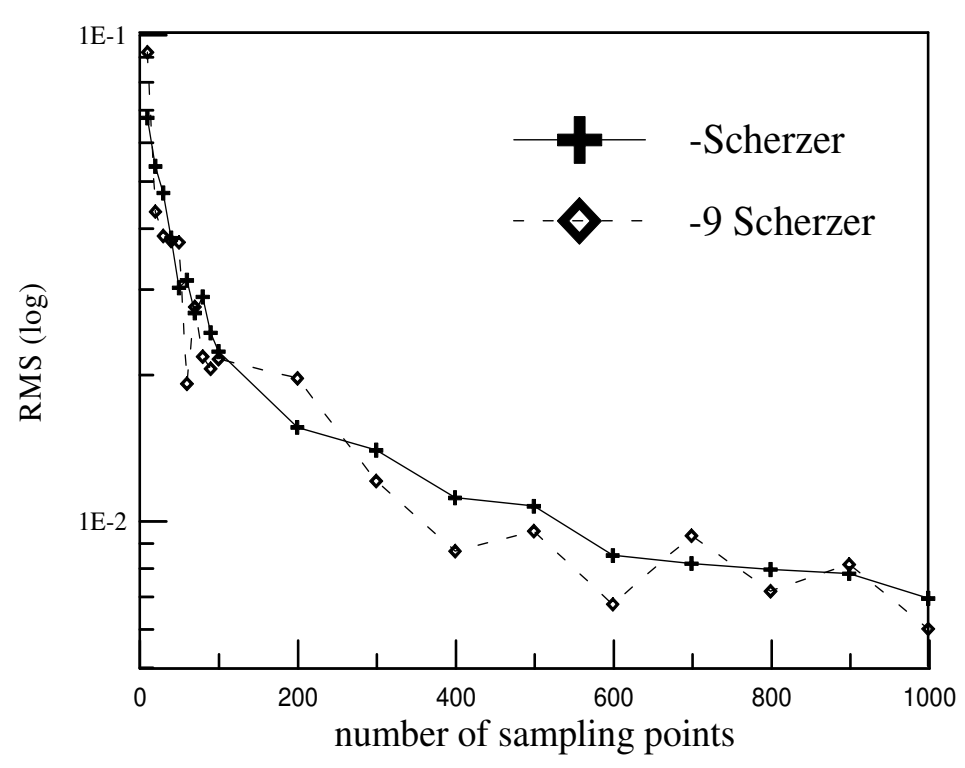

Fig. 1. RMS difference of image spectra calculated using the Monte Carlo method and the TCC for strong objects at defoci of Scherzer $(-344 \AA)$ and 9 Scherzer ($3094 \AA)$. The image spectra were simulated for an accelerating voltage of $300 \mathrm{kV}, \mathrm{C}_{3}=0.6 \mathrm{~mm}$, and Gaussian beam divergence and focal spread distributions, with $\mathrm{e}^{-1}$ values of $0.28 \mathrm{mrad}$, and $42 \AA$, respectively.

\section{Reference}

[1] W. O. Saxton, Scanning Microscopy 2 (1998) 213.

[2] A. I. Kirkland et al., Ultramicroscopy 15 (1995) 151

[3] D. Gabor, Nature 161 (1948) 777.

[4] M. Op de Beeck et al., Ultramicroscopy 64 (1996) 167.

[5] W. O. Saxton, Computer Processing of Electron Microscope Images, Springer Verlag, Berlin, 1980.

[6] E. J. Kirkland, Ultramicroscopy 9 (1982) 45.

[7] W. M. J. Coene et al, Ultramicroscopy 64 (1996) 109.

[8] M. Born and E. Wolf, Principles of Optics, 6th ed., Pergamon, Oxford,1980 . [9] K. Ishizuka, Ultramicroscopy 5 (1980) 5.

[10] W.H. Press, Numerical Recipes in C, $2^{\text {nd }}$ ed., Cambridge University Press, Cambridge, 1992.

[11] R. R. Meyer et al., Ultramicroscopy 85 (2000) 9. (a)

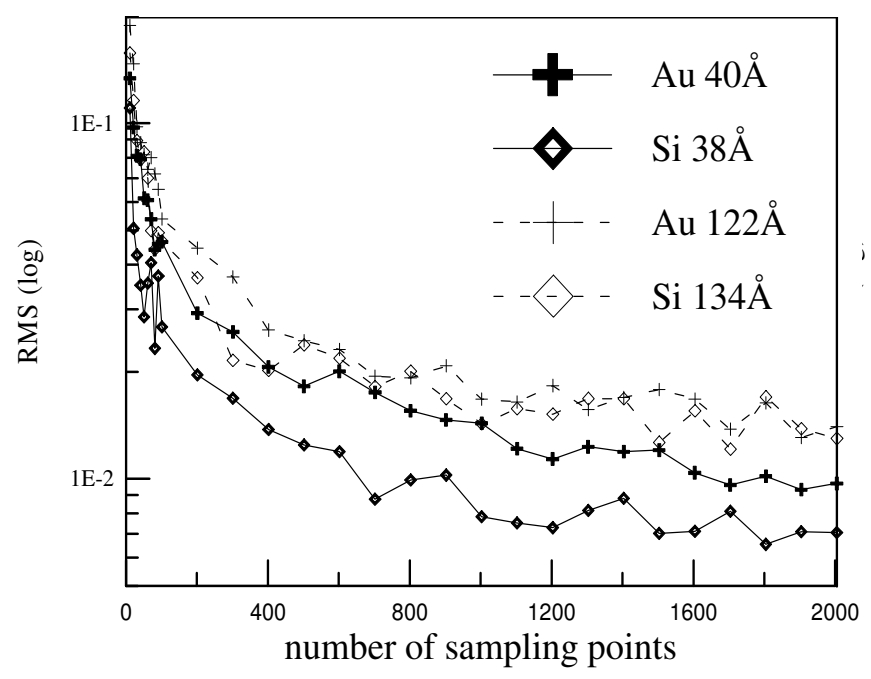

(b)

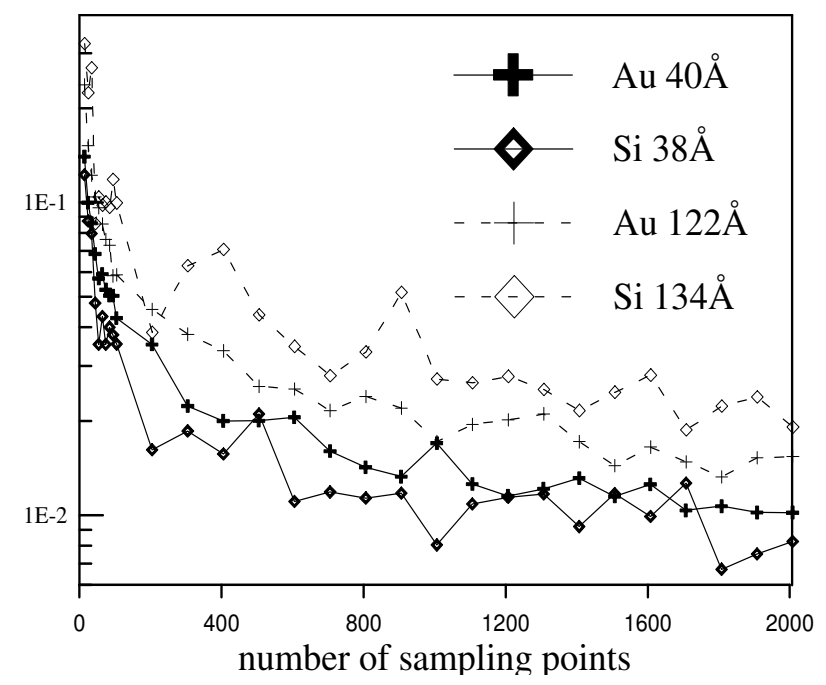

Fig. 2. RMS difference of image spectra calculated at (a) Scherzer and (b) 9 Scherzer defocus for Si [110] at thicknesses $38 \AA$ and $134 \AA$, and Au [100] at thicknesses $40 \AA$ and $122 \AA$. Other simulation parameters are the same as those in Fig. 1. 